\title{
FINE STRUCTURE OF GENES CONTROLLING PATHOGENICITY IN FLAX RUST, MELAMPSORA LINI
}

\author{
G. J. LAWRENCE*, K. W. SHEPHERD + and G. M. E. MAYO* \\ * Department of Genetics, The University of Adelaide, G.P.O. Box 498, Adelaide, South \\ Australia 5001; † Department of Agronomy, Waite Agricultural Research Institute, The \\ University of Adelaide, Glen Osmond, South Australia 5064.
}

Received 7.x.80

\begin{abstract}
SUMMARY
The fine-structure of four genes, $A-P, A-P 1, A-P 2$ and $A-P 3$ in the flax rust organism, Melampsora lini, that control pathogenicity on the host, Linum usitatissimum, has been investigated by using a mass-screening procedure to search for rare recombinant progeny. Three presumptive recombinant individuals were recovered from amongst 3160 dikaryotic test-cross progeny and seven from a second test in which haploid basidiospores were screened for recombinants. From the pathogenicity phenotypes of the presumptive recombinants, and their frequency of occurrence, it is concluded that genes $A-P, A-P 1, A-P 2$ and $A-P 3$ are sufficiently closely linked to belong to the same cistron. However, the possibility that they belong to separate, closely-linked cistrons can not be excluded: if they do belong to separate cistrons, then the data suggest that these are contiguous. A tentative gene order $A-P A-P 2 A-P 1 A-P 3$ is proposed.
\end{abstract}

\section{INTRODUCTION}

THE most comprehensive study of the genetics of the interaction that occurs between a plant host and its pathogen has been made with flax (Linum usitatissimum L.) and its rust (Melampsora lini (Ehrenb.) Lév.), due largely to the extensive work of Flor. In flax 29 genes have been identified so far that confer resistance to rust and for each of these host genes the pathogen appears to possess a specific and complementary gene conferring avirulence (Flor, 1956; 1965a; Lawrence et al. 1981). Host resistance is dominant to susceptibility and pathogen avirulence is usually dominant to virulence. The 29 host genes occur in five groups, with the genes within each group being located in the same small segment of host chromosome. These groups, designated $K, L, M, N$, and $P$, contain $1,13,7,3$ and 5 genes respectively. The structure of the genes within these groups has been investigated by screening for rare recombinants amongst large numbers (often thousands) of progeny from a plant heterozygous for two resistance genes from the same group (Shepherd, 1963; Flor, 1965b; Shepherd and Mayo, 1972; Mayo and Shepherd, 1980). The results of these studies have been interpreted as indicating that the genes in the $L$ group belong to the same cistron, that is, they are functional alleles, and that the genes in the $M$ and $N$ groups most likely belong to separate, closely-linked cistrons. With the $M$ genes these studies have also provided evidence as to the linear order of some of these genes (Mayo and Shepherd, 1980).

In comparison to the genes conferring resistance in the host, the corresponding avirulence genes in the pathogen exhibit less grouping. Only four groups of apparently tightly-linked genes have been found, namely 
A-L3* A-L4 A-L10, A-L5 A-L6 A-L7, A-M1 A-M4 and A-P A-P1 A-P2 $A-P 3$ (Flor, 1955; $1965 a$; Lawrence et al., 1981). So far, recombination between genes within these groups has not been reported. In studies of the inheritance of pathogenicity in flax rust, family sizes have always been small (less than 200), because testing individual rust cultures for their reactions on a range of host varieties is both time consuming and tedious. Therefore, it has been impractical to search for rare recombination between closelylinked genes controlling pathogenicity, where family sizes of several thousand would perhaps be needed. However, recently one of us (K.W.S.) devised a selection procedure that avoided the need to test individual progeny cultures and allowed large numbers of progeny to be screened for rare recombinants. This screening procedure was used in two ways to detect recombination between the four closely-linked avirulence genes $A-P, A-P 1$, $A-P 2$ and $A-P 3$. It was used first to screen dikaryotic test-cross progeny for recombinants and second, to screen haploid basidiospores and the results are reported in this paper.

\section{MATERIALS AND METHODS}

Strains $\mathrm{CH}_{5}$ and I were used in the test-cross. Strain $\mathrm{CH}_{5}$ is a hybrid strain derived from intercrossing strains $\mathrm{C}$ and $\mathrm{H}$. The origins of strains $\mathrm{C}, \mathrm{H}$ and I have been given previously (Lawrence et al., 1981). Strain I is fully virulent on host varieties Koto, Akmolinsk, Abyssinian and Leona, which possess genes $P, P 1, P 2$ and $P 3$ respectively, and hence it has the pathogenicity genotype a-P a-P1 a-P2 a-P3/a-P a-P1 a-P2 a-P3 (order arbitrary). Strain $\mathrm{CH}_{5}$ is avirulent on each of these host varieties. Since parent strain $C$ is avirulent on Koto $(P)$, fully virulent on Akmolinsk (P1) and Abyssinian $(P 2)$ and semi-virulent on Leona $(P 3)$ and strain $\mathrm{H}$ is virulent on Koto $(P)$ and avirulent on Akmolinsk $(P 1)$, Abyssinian $(P 2)$ and Leona $(P 3)$ it could be inferred that the genotype of strain $\mathrm{CH}_{5}$ is $A-P$ a-P1 a-P2 $a-P 3 / a-P A-P 1 A-P 2 A-P 3$ (order arbitrary). In fact, this arrangement of the genes in strain $\mathrm{CH}_{5}$, and their close linkage, was confirmed when 80 progeny cultures obtained by self-fertilizing strain $\mathrm{CH}_{5}$, and 32 cultures obtained by intercrossing strains $\mathrm{CH}_{5}$ and $\mathrm{I}$, were individually tested on the $P, P 1, P 2$ and $P 3$ host varieties (Lawrence et al., 1981).

The procedure used to detect rare recombination between the four " $A-P$ " genes depended on first making individual test-crosses between strain $\mathrm{CH}_{5}$, the multiple heterozygote, and strain I, the multiple recessive parent, to give test-cross progeny each consisting of a pustule of aeciospores. Spores from 60 to 110 of these test-cross progeny were then bulked and screened for recombinants by inoculating them on to a set of three tester-host stocks with genotypes $P P 1, P P 2$ and $P P 3$ which were obtained by crossing Koto $(P)$ to Akmolinsk (P1), Abyssinian (P2) and Leona (P3), respectively. The parental-type progeny from the test-cross will possess either gene $A-P$ or the gene combination $A-P 1 A-P 2 A-P 3$ and thus they will not grow on any of the three tester stocks. However, one half of the recombinants should be able to grow on one or more of these host stocks. For example, given the gene order above, a crossover between $A-P 1$ and $A-P 2$ in $\mathrm{CH}_{5}$ will produce

* In previous publications, following Flor, we have denoted host and pathogen genes by use of super- and sub-scripts. In this paper we have adopted a single line notation. For example, host gene $L^{3}$ is now denoted as $L 3$ and pathogen gene $A_{L^{3}}$ is now denoted as $A-L 3$. 
recombinant test-cross progeny with genotypes $a-P A-P 1$ a-P2 $a-P 3 / a-P$ a-P1 $a-P 2$ a-P3 and $A-P$ a-P1 A-P2 A-P3/a-P a-P1 a-P2 a-P3. The first type of recombinant will be detected by its growth on the $P P 2$ and $P P 3$ tester-host stocks. The second type, however, will not grow on any of the tester-host stocks and therefore it will not be detected. Thus the screening procedure possesses the limitation that it will detect only half of the expected recombinant progeny.

A single fertilized pycnium of flax rust produces a pustule containing numerous aeciospores. Therefore, when the aeciospores collected from many pustules are well mixed prior to inoculation, we can expect that inclusion of just one recombinant pustule in the bulk sample will result in the occurrence of multiple uredospore pustules on the susceptible host(s).

Strain $\mathrm{CH}_{5}$ was used as the "recipient" parent in all crosses since aeciospores arising from accidental selfing of strain $\mathrm{CH}_{5}$ will not grow on any of the tester-host stocks. On the other hand, if strain I had been used as the recipient parent, accidental selfings would have produced aeciospores virulent on all three tester-host stocks, and these could have been misclassified as recombinants.

To make the crosses, plants of the "universally susceptible" variety Hoshangabad were first inoculated with haploid basidiospores by suspending germinating teliospores of a parent strain over the plants for several hours in a humidity chamber. Eight to ten days after inoculation monokaryotic infections (pycnia) appear and these produce pycniospores in a liquid exudate (nectar). To prevent uncontrolled crosses all multiplyinfected leaves were removed one to two days before the appearance of nectar and the plants were protected from insects by placing them under frames covered with clear cellophane. Approximately five days after the first appearance of nectar, crosses were made by transferring nectar from strain I pycnia to $\mathrm{CH}_{5}$ pycnia. Nectar from about 20-25 pycnia of strain I was collected and added to $0.6 \mathrm{ml}$ of tap water in a small glass phial, and a drop of this diluted nectar was transferred to a recipient pycnium with the aid of a plastic toothpick. A different toothpick was used for each transfer. From 120-140 pycnia of $\mathrm{CH}_{5}$ could be fertilized with one batch of $0.6 \mathrm{ml}$ nectar solution in about two hours. There was no evidence that dilution in water for two hours reduced the ability of pycniospores to effect fertilization.

Aeciospores arising from successful crosses were collected on water in a watch glass after the leaf containing the pustule had been cut from the plant. Usually aeciospores from about 60-110 pustules were collected and well mixed in the one watch glass, before being inoculated by brush on to three plants of each of the three tester-host stocks and on to Hoshangabad. The uredospores that developed on Hoshangabad were kept as a stock culture for further testing if required. Most of the uredospore pustules that developed on the tester-host stocks were increased separately on Hoshangabad and then tested on the four host differentials Koto $(P)$, Akmolinsk $(P 1)$, Abyssinian $(P 2)$ and Leona $(P 3)$ and sometimes on a set of 29 host differentials as well.

In the other approach used to isolate recombinants, basidiospores of strain $\mathrm{CH}_{5}$ were shed directly on to tester-host stocks of genotypes $P P 1, P P 2$ and $P P 3$ on the expectation that only recombinant basidiospores lacking the complementary avirulence genes would grow. To achieve a heavy inoculation, each pot of the tester stocks was inoculated for a period of eight to ten 
hours with basidiospores shed from one or two pieces of teliospore-bearing stem each two to three $\mathrm{cm}$ long. The position of the teliospores above the plants was changed at hourly intervals to ensure an even inoculation.

Scoring for pathogenicity reactions was carried out approximately 14 days after inoculation and was based on the amount of growth made by the rust on the host plant. Growth ranged from free $(+)$ to intermediate $(+/-$ and -$)$ to nil $(--)$. Full virulence $(+)$ was characterized by large, often compound, uredospore pustules on the inoculated leaves (both young and old) and stems, whereas avirulence ( - ) had necrotic flecks on inoculated leaves with no sign of pustule development. Semi-virulent reactions showed restricted pustule development with the extent of restriction usually dependent on the age of the leaf. When small $(0.5 \mathrm{~mm}$ diameter $)$ pustules developed on the very young leaves, but no pustules occurred on older leaves, the reaction was scored as - . When there was only slight restriction of pustule development on young leaves and markedly smaller or no pustules on the lower, older leaves the reaction was scored as $+/-$. Reactions intermediate between these categories were scored as $-(+/-)$ or $+/-(+)$.

\section{RESUlts}

\section{(i) Screening dikaryotic test-cross progeny for recombinants}

Nectar from strain I pycnia was transferred to 5073 individual pycnia of strain $\mathrm{CH}_{5}$ distributed over 97 pots of Hoshangabad plants and $3160(62 \cdot 3$ per cent) of these pycnia produced aeciospores. This percentage success was much less than expected, since the strain I nectar used was a mixture collected from at least 20 pycnia, so that the mixture should always have contained pycniospores of different mating type from that of the recipient pycnium. Furthermore, the mating-type specificities of strain I pycnia are known to differ from those of strain $\mathrm{CH}_{5}$ pycnia (Lawrence, 1980). The low success rate is attributed to suboptimal growth conditions because, due to a shortage of controlled environment space, most plants had to be kept on a laboratory bench adjacent to a window, where the light and temperature was often below that required for successful fertilization and aeciospore development. A success rate of less than 50 per cent was common on these plants in winter, whereas in spring the success rate frequently approached 100 per cent.

The 3160 test-cross progeny were grouped into 39 bulk collections and these mixtures were separately inoculated on to the tester-host stocks to screen for recombinants. With nine collections, no uredospore pustules developed on any of the tester-host stocks, whereas with the other 30 collections, one or more pustules arose on at least one of the three tester stocks. As shown in table 1, these pustules could be classified into one of four different pathogenicity phenotypes when tested on the host varieties Koto $(P)$, Akmolinsk (P1), Abyssinian (P2) and Leona (P3).

\section{(a) Pustules of phenotype class 1}

Uredospore pustules possessing phenotype 1 all arose as minute pustules on the youngest leaves of the PP3 tester-host and, on testing, proved to be virulent on Koto $(P)$, but avirulent on A kmolinsk $(P 1)$, Abyssinian $(P 2)$ and 
TABLE 1

Pathogenicity phenotypes of uredospore pustules recovered after inoculating bulk collections of test-cross rust progeny on tester-host stocks

\begin{tabular}{|c|c|c|c|c|c|c|c|c|}
\hline \multirow[t]{2}{*}{$\begin{array}{l}\text { Phenotype } \\
\text { class }\end{array}$} & \multirow[t]{2}{*}{$\begin{array}{l}\text { Bulk } \\
\text { collection } \\
\text { no. }\end{array}$} & \multicolumn{3}{|c|}{$\begin{array}{l}\text { Number of uredospore } \\
\text { pustules recovered on } \\
\text { tester-host stocks* }\end{array}$} & \multicolumn{4}{|c|}{ Pathogenicity $\dagger$ on host variety } \\
\hline & & $P P 1$ & $P P 2$ & $P P 3$ & $\begin{array}{c}\text { Koto } \\
(P)\end{array}$ & $\begin{array}{c}\text { Akmol. } \\
\text { (P1) }\end{array}$ & $\begin{array}{l}\text { Abys. } \\
(P 2)\end{array}$ & $\begin{array}{c}\text { Leona } \\
\text { (P3) }\end{array}$ \\
\hline 1 & $\begin{array}{r}4 \\
6 \\
8 \\
10 \\
13 \\
14 \\
15 \\
21 \\
28 \\
38\end{array}$ & $\begin{array}{l}0 \\
0 \\
0 \\
0 \\
0 \\
0 \\
0 \\
0 \\
0 \\
0\end{array}$ & $\begin{array}{l}0 \\
0 \\
0 \\
0 \\
0 \\
0 \\
0 \\
0 \\
0 \\
0\end{array}$ & $\begin{array}{l}2 \\
1 \\
1 \\
1 \\
1 \\
2 \\
1 \\
1 \\
1 \\
1\end{array}$ & $\begin{array}{l}+ \\
+ \\
+ \\
+ \\
+ \\
+ \\
+ \\
+ \\
+ \\
+\end{array}$ & $\begin{array}{l}-- \\
-- \\
-- \\
-- \\
-- \\
-- \\
-- \\
-- \\
-- \\
--\end{array}$ & $\begin{array}{l}-- \\
-- \\
-- \\
-- \\
-- \\
-- \\
-- \\
-- \\
-- \\
--\end{array}$ & $\begin{array}{l}-- \\
-- \\
-- \\
-- \\
-- \\
-- \\
-- \\
-- \\
-- \\
--\end{array}$ \\
\hline 2 & $\begin{array}{l}17 \\
21 \\
23 \\
25\end{array}$ & $\begin{array}{l}1 \\
1 \\
1 \\
1\end{array}$ & $\begin{array}{l}0 \\
0 \\
0 \\
0\end{array}$ & $\begin{array}{l}0 \\
0 \\
0 \\
0\end{array}$ & $\begin{array}{l}+ \\
+ \\
+ \\
+\end{array}$ & $\begin{array}{l}+ \\
+ \\
+ \\
+\end{array}$ & $\begin{array}{l}-- \\
-- \\
-- \\
--\end{array}$ & $\begin{array}{l}-- \\
-- \\
-- \\
--\end{array}$ \\
\hline 3 & 7 & 3 & 0 & 8 & + & - & -- & $+1-$ \\
\hline $4 a$ & $\begin{array}{l}19 \\
36\end{array}$ & $\begin{array}{l}10 \\
20(9)\end{array}$ & $\begin{array}{c}7 \\
32(9)\end{array}$ & $\begin{array}{c}8 \\
28(9)\end{array}$ & $\begin{array}{l}+ \\
+\end{array}$ & $\begin{array}{l}+ \\
+\end{array}$ & $\begin{array}{l}+ \\
+\end{array}$ & $\begin{array}{l}+ \\
+\end{array}$ \\
\hline$b$ & $\begin{array}{r}1 \\
2 \\
3 \\
4 \\
10 \\
11 \\
13 \\
15 \\
16 \\
17 \\
18 \\
21 \\
22 \\
27 \\
28 \\
29 \\
30 \\
31 \\
32 \\
33 \\
37\end{array}$ & $\begin{array}{l}0 \\
0 \\
0 \\
1 \\
1 \\
1 \\
1 \\
0 \\
0 \\
1 \\
2 \\
0 \\
1 \\
4 \\
2 \\
1 \\
4 \\
2 \\
1 \\
1 \\
1\end{array}$ & $\begin{array}{c}0 \\
1 \\
1 \\
0 \\
2 \\
2 \\
0 \\
1 \\
1 \\
3 \\
1 \\
1 \\
0 \\
2 \\
9 \\
3 \\
15(8) \\
9(7) \\
1 \\
0 \\
2\end{array}$ & $\begin{array}{l}1 \\
0 \\
0 \\
0 \\
0 \\
0 \\
0 \\
1 \\
0 \\
1 \\
0 \\
0 \\
0 \\
0 \\
1 \\
0 \\
1 \\
1 \\
0 \\
0 \\
2\end{array}$ & $\begin{array}{l}+ \\
+ \\
+ \\
+ \\
+ \\
+ \\
+ \\
+ \\
+ \\
+ \\
+ \\
+ \\
+ \\
+ \\
+ \\
+ \\
+ \\
+ \\
+ \\
+ \\
+\end{array}$ & $\begin{array}{l}+ \\
+ \\
+ \\
+ \\
+ \\
+ \\
+ \\
+ \\
+ \\
+ \\
+ \\
+ \\
+ \\
+ \\
+ \\
+ \\
+ \\
+ \\
+ \\
+\end{array}$ & $\begin{array}{l}+ \\
+ \\
+ \\
+ \\
+ \\
+ \\
+ \\
+ \\
+ \\
+ \\
+ \\
+ \\
+ \\
+ \\
+ \\
+ \\
+ \\
+ \\
+ \\
+ \\
+\end{array}$ & $\begin{array}{l}+ \\
+ \\
+ \\
+ \\
+ \\
+ \\
+ \\
+ \\
+ \\
+ \\
+ \\
+ \\
+ \\
+ \\
+ \\
+ \\
+ \\
+ \\
+ \\
+ \\
+\end{array}$ \\
\hline $4 b$ & totals & 24 & 54 & 8 & & & & \\
\hline
\end{tabular}

* All the pustules recovered were tested for phenotype except where the number was large and then the number tested is shown in parentheses.

$\dagger+=$ full growth of rust; $+/-=$ restricted growth; $-=$ minute pustules on youngest leaves only; $--=$ no growth.

Leona $(P 3)$ (see table 1). These pustules are almost certainly parental products that have achieved limited growth on the PP3 tester because the Leona gene, $P 3$, is not always completely effective in suppressing the growth of an "avirulent" strain, particularly on the youngest leaves. The presence 
of only a single dose of the $P 3$ gene in the tester-host and only a single dose of the $A-P 3$ gene in the rust may have accentuated this "leakiness".

\section{(b) Pustules of phenotype class 2}

The reaction pattern of the four pustules of phenotype 2 (table 1 ) on the four " $P$ " gene hosts and on a set of 29 host differentials matched that of another strain being used concurrently in another programme. Since these reactions are incompatible with either a $\mathrm{CH}_{5}$-selfed, I-selfed or $\mathrm{CH}_{5} \times \mathrm{I}$ origin, these pustules have been classified as contaminants.

\section{(c) Pustules of phenotype class 3}

The 11 pustules of phenotype 3 most likely have a common origin, since they all arose within one collection (see table 1 ) and they possess a reaction pattern on the $P, P 1, P 2$ and $P 3$ differentials that has not been recorded before. Apparently these pustules represent clonal progeny from a single fertilized pycnium of $\mathrm{CH}_{5}$ that was derived from a basidiospore having a non-parental combination of the $A-P, A-P 1, A-P 2$ and $A-P 3$ genes. The most likely cause of this new gene combination is recombination within the segment containing the " $A-P$ " genes, but the possibility of a mutation cannot be eliminated. One of the 11 pustules was tested on a set of 29 host differentials and its reactions were consistent with it having originated from the test-cross, $\mathrm{CH}_{5} \times \mathrm{I}$.

The three pustules detected on the PP1 tester-host were extremely restricted in growth and the eight detected on the $P P 3$ tester-host were moderately restricted. This restriction in growth was due to the $P 1$ and $P 3$ genes, respectively, since these isolates grew freely when tested on Koto $(P)$, but showed restricted growth on Akmolinsk (P1) and Leona (P3) (see table 1).

\section{(d) Pustules of phenotype class 4}

Pustules virulent on all four of the single " $P$ " gene differentials were recovered from 23 out of 39 collections (table 1). Two of the 23 collections (No. 19 and No. 36) produced a large number of uredospore pustules in approximately equal numbers on all three of the tester-host stocks (see table 1 , phenotype $4 \mathrm{a}$ ) whereas the pustules recovered from the other collections (table 1, phenotype 4b) were either very few in number or, if numerous, did not arise with equal frequency on each of the tester-host stocks; there was a consistent tendency for these pustules to arise frequently on $P P 2$, less frequently on $P P 1$ and only rarely on $P P 3$.

These two dissimilar patterns of pustule distribution suggested that more than one mechanism might be responsible for the observed variants. Besides meiotic recombination, it is necessary to consider whether some of these pustules were derived, in some way, solely from the test-cross parent, strain I, since, at the time of these tests, this was the only strain held at Adelaide able to attack the four host genes $P, P 1, P 2$ and $P 3$. Unfortunately it is difficult to distinguish strain I derivatives from $\mathrm{CH}_{5} \times \mathrm{I}$ derivatives because in tests on a set of 29 differentials the progeny from both these families segregated on the same differentials and had identical reactions on those 
differentials on which segregation did not occur except for the $P, P 1, P 2$ and $P 3$ differentials and Bolley Golden selection $(L 1 O)$ (see Lawrence et al., 1981). Thus the reaction on Bolley Golden selection provided the only possibility for discriminating between the two possible parentages of the pustules since the I-selfed progeny were all avirulent on Bolley Golden selection, whereas the $\mathrm{CH}_{5} \times \mathrm{I}$ progeny segregated for virulence and avirulence in approximately equal numbers. Thus, if a pustule was virulent on Bolley Golden selection, this would be an indication of a $\mathrm{CH}_{5} \times \mathrm{I}$ origin; a pustule avirulent on Bolley Golden selection could have either I-selfed or $\mathrm{CH}_{5} \times$ I parentage.

Since progeny from both $\mathrm{CH}_{5} \times \mathrm{I}$ and I-selfed parentage segregated on a number of differential varieties in the family studies (Lawrence et al., 1981), it should be possible to determine whether all pustules of phenotype class 4 recovered from a given bulk collection come from the same or separate events. If pustules were derived from separate events they would be expected to frequently possess different pathogenicity reactions on some of the differentials, irrespective of whether they came from $\mathrm{CH}_{5} \times \mathrm{I}$ crosses or I-selfed parentage.

Therefore, to test for both the parentage of the phenotype class 4 pustules and for whether pustules recovered from the same collection were derived from the same or separate events, 42 uredospore pustules of phenotype class 4 from eight bulk collections of aeciospores were each tested on a set of 29 host differentials and the results are given in table 2 . The

TABLE 2

Pathogenicity of a sample of pustules of phenotype class 4 on a set of 29 host differentials

\begin{tabular}{|c|c|c|c|c|}
\hline $\begin{array}{c}\text { Phenotype } \\
\text { class }\end{array}$ & $\begin{array}{l}\text { Bulk } \\
\text { collection } \\
\text { no. }\end{array}$ & $\begin{array}{c}\text { Number of pustules } \\
\text { tested on set of } \\
\text { differentials }\end{array}$ & $\begin{array}{l}\text { Reaction } \\
\text { patterns }\end{array}$ & $\begin{array}{l}\text { Reaction on } \\
\text { Bolley Golden } \\
\text { selection }\end{array}$ \\
\hline $4(a)$ & $\begin{array}{l}36 \\
19\end{array}$ & $\begin{array}{l}9 \\
9\end{array}$ & $\begin{array}{l}\text { all same } \\
\text { all same }\end{array}$ & $\begin{array}{l}\text { all + } \\
\text { all - }\end{array}$ \\
\hline $4(b)$ & $\begin{array}{l}17 \\
27 \\
28 \\
30 \\
31 \\
37\end{array}$ & $\begin{array}{l}4 \\
4 \\
4 \\
4 \\
4 \\
4\end{array}$ & $\begin{array}{l}2 \text { different } \\
4 \text { different } \\
4 \text { different } \\
3 \text { different } \\
4 \text { different } \\
3 \text { different }\end{array}$ & $\begin{array}{l}\text { all - } \\
\text { all - } \\
\text { all - } \\
\text { all - } \\
\text { all - } \\
\text { all - }\end{array}$ \\
\hline
\end{tabular}

* 2 pustules had one pattern and 2 had another.

$++=$ pustules virulent; $-=$ pustules avirulent.

nine pustules tested from collection No. 36 all had identical reaction patterns and were virulent on Bolley Golden selection, suggesting a common origin from a $\mathrm{CH}_{5} \times$ I cross. It is likely they all represent clonal progeny from a single fertilized pycnium of $\mathrm{CH}_{5}$ that possessed the four virulent genes $a-P, a-P 1, a-P 2$ and $a-P 3$, most likely as a result of rare recombination, but again mutation cannot be excluded. The nine pustules from collection No. 19 also had identical reaction patterns, suggesting that they were similarly derived from a single fertilized pycnium possessing $a-P, a-P 1$, $a-P 2$ and $a-P 3$. Again, this genotype most likely arose from recombination or mutation in $\mathrm{CH}_{5}$. However, since the pustules were all avirulent on 
Bolley Golden selection the possibility of their origin from I-selfed cannot be excluded.

Since all pustules from each of collections Nos. 19 and 36 gave identical reaction patterns, it is concluded that in each case they represent clonal products of a single fertilization event. If multiple fertilization of a single pycnium had occurred in these crosses, it would be expected that diverse reaction patterns would have been found amongst the pustules recovered, since the nectar used in the crosses was a mixture collected from numerous pycnia of strain I. Multiple fertilization within a pycnium is known to occur in some rust species (see Dinoor et al., 1968), but it has not been reported in flax rust.

In contrast to the above results, the uredospore pustules recovered from collections Nos. 17, 27, 28, 30, 31 and 37 mostly had different reaction patterns (table 2), thus indicating that these at least arose from separate events. Further, all 24 pustules tested were avirulent on Bolley Golden selection (table 2), which makes it unlikely that they came from $\mathrm{CH}_{5} \times \mathrm{I}$ crosses since 50 per cent of such cultures are expected to be virulent on Bolley Golden selection. Therefore it is probable that most, if not all, of these pustules were derived from strain I and not the test-cross. It is likely that the untested pustules from the other 15 collections listed under phenotype $4 \mathrm{~b}$, table 1 , are of similar origin since few pustules were recovered from within each of these collections (in contrast to the collections listed under phenotype $4 \mathrm{a}$, table 1) and there was a consistent tendency for the pustules to arise most frequently on the $P P 2$ tester-host, less frequently on $P P 1$ and only rarely on $P P 3$, just as observed with the six collections from which pustules were tested (collections Nos. 17, 27, 28, 30, 31, 37).

This unequal frequency of recovery of pustules of the $4 \mathrm{~b}$ type on the three different tester hosts probably has special significance because there is evidence which indicates that the three tester-host stocks are equally efficient at screening out rare virulent spores present in an inoculum of avirulent spores. Thus collections Nos. 19 and 36, which each appear to have contained just one pustule of virulent aeciospores, gave approximately equal numbers of uredospore pustules on each of the three testers (table 1). As a further test of the relative efficiency of the different testers to detect rare virulent pustules, three control screenings were carried out. Three batches of inoculum were prepared, each consisting of one pustule of strain I uredospores (virulent) added to 80 pustules of $\mathrm{CH}_{5}$ uredospores (avirulent). Each batch of inoculum was then applied to three, $20 \mathrm{~cm}$ tall plants in one pot of each of the three tester-host stocks $P P 1, P P 2$ and $P P 3$. In all three trials approximately equal numbers of pustules appeared on each of the tester stocks, again indicating that these different stocks possess equivalent screening abilities.

Since the type $4 \mathrm{~b}$ uredospore pustules were not recovered in equal frequencies on all three testers, it is likely that these pustules did not arise from virulent spores present in the original inoculum. Rather, these pustules may have been derived from virulent cells that arose after inoculation, and possible mechanisms for this will be considered in the discussion. In summary, therefore, it is likely that the phenotype 4 pustules that arose in collections Nos. 19 and 36 were each derived from a single recombinant or mutant gamete of $\mathrm{CH}_{5}$, whereas the pustules from collections listed under 
$4 \mathrm{~b}$ (table 1) appear to be derived entirely from strain I and probably arose after inoculation.

\section{(ii) Screening basidiospores for recombinants}

A second method of screening for recombinants involved direct inoculation of the three tester-host stocks $P P 1, P P 2$ and $P P 3$ with basidiospores shed from germinating teliospores of $\mathrm{CH}_{5}$ (genotype a-P A-P1 A-P2 $A-P 3 / A-P$ a-P1 a-P2 a-P3). Basidiospores possessing a parental genotype ( $a-P A-P 1 A-P 2 A-P 3$ or $A-P a-P 1$ a-P2 $a-P 3$ ) should not grow on any of these tester stocks, whereas half of the recombinant basidiospores should be capable of growing on one or more of the testers: providing that the same avirulence genes function in the monokaryon as in the dikaryon. Flor (1959a) and Statler and Gold (1980) have demonstrated that many avirulence genes, including $A-P, A-P 1, A-P 2$ and $A-P 3$, will prevent monokaryon growth on host lines possessing the complementary genes for resistance.

To test whether the $A-P, A-P 1, A-P 2$ and $A-P 3$ genes in $\mathrm{CH}_{5}$ also function in the monokaryon, the host varieties Koto $(P)$, Akmolinsk $(P 1)$, Abyssinian $(P 2)$ and Leona $(P 3)$ were inoculated with basidiospores from $\mathrm{CH}_{5}$. The pycnia that arose on a given host variety were intercrossed and the resultant progeny cultures were tested for pathogenicity. Progeny derived from pycnia on Koto $(P)$ were always virulent on Koto and progeny derived from pycnia on Akmolinsk (P1) and Abyssinian (P2) were always virulent on these host varieties, thus indicating that the $A-P, A-P 1$ and $A-P 2$ genes in $\mathrm{CH}_{5}$ do function in the monokaryon. Although a few, very small, pycnia developed on Leona in these tests, no aeciospores developed when they were intercrossed. This result was not unexpected, for the $a-P 3$ gene in $\mathrm{CH}_{5}$ only confers semi-virulence to Leona $(P 3)$ in the dikaryon (Lawrence et al., 1981). It appears that the avirulence allele $(A-P 3)$ in $\mathrm{CH}_{5}$ prevents, and the semi-virulence allele ( $a-P 3)$ severely restricts basidiospore (monokaryon) growth on Leona (P3). Thus these preliminary studies indicated that parental-type basidiospores will not grow on any of the tester-host stocks, and therefore it should be feasible to screen directly for recombinant basidiospores of $\mathrm{CH}_{5}$. However, since the semi-virulence allele $a-P 3$ also seems to restrict monokaryon growth on a host possessing the $P 3$ gene, this basidiospore-screening approach may not recover some types of recombinants that would be recovered from screening the dikaryotic testcross progeny.

In the first screening, basidiospores of $\mathrm{CH}_{5}$ were inoculated on to 33 pots of each of the tester-host plants PP1, PP2 and PP3 but no pycnia developed (table 3, trial 1). However, it was noted that the leaves of the tester-host plants were "harder" than those of the variety Hoshangabad and it was thought that the failure to obtain pycnia may have been due to this leaf "hardness" rather than to the absence of recombinants. Therefore new tester stocks were constructed in which 50 per cent of the background genotype was of Hoshangabad origin by backcrossing to Hoshangabad. In a second test 14 pots of each of these stocks were inoculated with $\mathrm{CH}_{5}$ basidiospores, but again no pycnia were recovered (table 3 , trial 2). 
Later a third attempt was made using tester stocks in which $7 / 8$ of the background genotype was of Hoshangabad origin. Thirty-seven pots of each tester were inoculated and seven pycnia developed on these plants (table 3, trial 3). Nectar from each of these pycnia was used to fertilize several pycnia of strain I, after which each pycnium on the tester plants was itself fertilized by a mixture of strain I nectar. Aeciospores arising from these crosses were increased on Hoshangabad before testing on the host differentials.

\section{TABLE 3}

Number of pycnia recovered on tester-host stocks, with varying proportions of Hoshangabad genotype, after inoculation with basidiospores of strain $\mathrm{CH}_{5}$

\begin{tabular}{|c|c|c|c|c|c|}
\hline \multirow[t]{2}{*}{$\begin{array}{c}\text { Trial } \\
\text { no. }\end{array}$} & \multirow[t]{2}{*}{$\begin{array}{l}\text { Number of pots } \\
\text { of each tester } \\
\text { inoculated }\end{array}$} & \multirow[t]{2}{*}{$\begin{array}{c}\text { Proportion of } \\
\text { Hoshangabad } \\
\text { genotype in } \\
\text { tester stocks }\end{array}$} & \multicolumn{3}{|c|}{$\begin{array}{l}\text { Number of pycnia } \\
\text { arising on } \\
\text { tester-host stock }\end{array}$} \\
\hline & & & $P P 1$ & $P P 2$ & $P P 3$ \\
\hline 1 & 33 & 0 & 0 & 0 & 0 \\
\hline 2 & 14 & $\frac{1}{2}$ & 0 & 0 & 0 \\
\hline 3 & 37 & $\frac{7}{8}$ & 3 & 2 & 2 \\
\hline
\end{tabular}

The cultures derived from six of the seven presumptive recombinant pycnia were found on testing to be fully virulent on Koto $(P)$, Akmolinsk $(P 1)$, Abyssinian (P2) and Leona (P3). The remaining pycnium, which arose on the $P P 1$ tester, was very small and produced so little nectar that it was used in only one cross. The single culture arising from this cross was fully virulent on Koto, Akmolinsk and Leona but avirulent on Abyssinian $(P 2)$. This culture, and several derived from each of the other six pycnia, were also tested on a set of 29 host differentials. All had reaction patterns consistent with $\mathrm{a} \mathrm{CH}_{5} \times \mathrm{I}$ origin. In particular, these tests showed that five of the six pycnia that produced cultures virulent on Koto, Akmolinsk, Abyssinian and Leona could not be strain I contaminants, since the progeny derived from these five pycnia were all virulent on Bolley Golden selection, whereas I $\times$ I cultures are always avirulent on Bolley Golden selection (Lawrence et al., 1981). Since $\mathrm{CH}_{5} \times \mathrm{I}$ progeny have a 50 per cent probability of being avirulent on Bolley Golden selection (Lawrence et al., 1981), the remaining pycnium is also consistent with a $\mathrm{CH}_{5}$ origin. Additional evidence that the six pycnia that produced cultures virulent on Koto, Akmolinsk, Abyssinian and Leona are not strain I contaminants comes from the results of crossing these six pycnia to strain I pycnia. In all, 42 pycnia of strain I were fertilized by individual transfer of nectar from one or other of the six pycnia and 41 of these crosses produced aeciospores. This indicates that the six pycnia must have come from strain $\mathrm{CH}_{5}$ and not strain I contamination, because crosses between pyncia of strain $\mathrm{CH}_{5}$ and those of strain I are known to give aeciospores in nearly every instance, whereas less than 50 per cent of crosses between two pycnia of strain I origin give rise to aeciospores (Lawrence, 1980). Thus the seven pycnia recovered from screening basidiospores are almost certainly all of $\mathrm{CH}_{5}$ origin. 


\section{Discussion}

\section{(i) Significance of presumptive recombinant or mutant cultures}

The phenotypes of the ten variant cultures recovered from screening $\mathrm{CH}_{5} \times \mathrm{I}$ test-cross progeny and $\mathrm{CH}_{5}$ basidiospores are shown in table 4 , together with the phenotypes of the parent rust strains. Cultures Nos. 1 and 4 have reaction patterns that have not been reported previously. Six of the eight cultures that are virulent on all four of the host varieties possessing the $P, P 1, P 2$ and $P 3$ genes are also virulent on Bolley Golden selection and thus cannot be derived from contamination with strain I. Therefore it is considered that the ten variant cultures are each derived from a basidiospore of $\mathrm{CH}_{5}$ that possessed a non-parental combination of the $A-P, A-P 1, A-P 2$ and $A-P 3$ genes due to either mutation or meiotic recombination.

TABLE 4

Pathogenicity of ten presumptive recombinant or mutant cultures, and their parents, on host varieties Koto, Akmolinsk, Abyssinian and Leona

\section{Rust strain}

Parent $1(\mathrm{C})$

Parent $2(\mathrm{H})$

Hybrid $\left(\mathrm{CH}_{5}\right)$

Test-cross parent (I)

Recombinant (or mutant) cultures

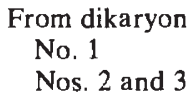

From monokaryon

No. 4

Nos. 5 to 10
Pathogenicity* on host variety

Koto $(P) \quad$ Akmolinsk $(P 1) \quad$ Abyssinian $(P 2)$

$\begin{array}{cc}-- & + \\ + & -- \\ -- & -- \\ + & +\end{array}$

$*+=$ full growth of rust; $+/-=$ restricted growth; $-=$ minute pustules on youngest leaves only; $--=$ no growth.

The high frequency of recovery of the $a-P a-P 1 a-P 2 a-P 3$ non-parental genotype compared to the other two non-parental genotypes probably reflects, at least partially, the bias introduced by the basidiospore screening test, from which seven of the ten non-parental variants were recovered. Statistically basidiospores capable of growing on all three tester-host stocks are more likely to be recovered in the screening test than those capable of growing on only one or two of the tester stocks. Furthermore, the nonparental combination of genes present in culture No. 1 from the test-cross (table 4) would not have been recovered on any of the tester stocks in the basidiospore screening since $A-P 1$ and $A-P 2$ would prevent growth on the $P P 1$ and $P P 2$ tester stocks, respectively, and the semi-virulence allele, $a-P 3$, as discussed above, would prevent monokaryon growth on host testers possessing $P 3$.

A feature of interest with these ten variant cultures is that, besides possessing a non-parental genotype each also shows a pathogenicity reaction on one of the four host differentials that is unlike any of the parental reactions (table 4). Thus culture No. 1 shows a small amount of growth on Akmolinsk, giving small pustules on the upper, younger leaves of the plant 
(scored as " - "), whereas the two parents of $\mathrm{CH}_{5}$, strains $\mathrm{C}$ and $\mathrm{H}$, are fully virulent $(+)$ and fully avirulent $(--)$, respectively, on Akmolinsk. The remaining nine cultures are fully virulent $(+)$ on Leona whereas strains $C$ and $\mathrm{H}$ are semi-virulent $(+/-(-))$ and avirulent $(--)$, respectively (table 4). These altered pathogenicity reactions are not likely to be due to segregating modifier genes, because such reactions were not shown by any of the 32 progeny cultures of $\mathrm{CH}_{5} \times \mathrm{I}$ origin tested in a previous study (Lawrence et al., 1981). It would seem, therefore, that the event which gave rise to the non-parental genotype in each of the ten cultures has also altered the pathogenicity reaction on one of the " $P$ " gene hosts.

Although it is not possible to decide unequivocally at the present time whether the ten variant cultures listed in table 4 are each derived from a mutational event or from meiotic recombination, there are two reasons why it is likely that most of them are derived from meiotic recombination. First, it is expected that recombinational events will usually be more frequent than mutational events. Second, in the present study, where meiotic recombination is a possibility, two variants were recovered that possessed novel genotypes (cultures Nos. 1 and 4, table 4). By contrast, in a screening test for somatic variants in which uredospores of strains $\mathrm{CH}_{5}$ (genotype $A-P$ a-P1 $a-P 2 a-P 3 / a-P A-P 1 A-P 2 A-P 3)$ and of two related strains of similar genotype $\left(\mathrm{CH}_{4}\right.$ and $\left.\mathrm{CH}_{6}\right)$ were inoculated on to the $P, P 1, P 2$ and $P 3$ host differentials, variants with novel genotypes were not recovered (Lawrence, 1977): the only variants obtained were those which had "lost" all avirulence specificities in the one strand and thus resembled the phenotypes of parent strain $\mathrm{C}$ or $\mathrm{H}$ on the " $P$ " gene differentials.

If the ten variant cultures are recombinants, as seems likely, the altered pathogenicity of culture No. 1 on Akmolinsk ( $P 1)$ and cultures Nos. 2-10 on Leona $(P 3)$ suggests that the recombination event from which culture No. 1 was derived occurred, at least partly, within the $A-P 1$ cistron and the events from which cultures Nos. 2-10 were derived occurred, at least partly, within the $A-P 3$ cistron. Thus, if the assumption of a recombinational origin is correct, then events that recombine $A-P, A-P 1, A-P 2$ and $A-P 3$ apparently always occur within these genes, which suggests that if $A-P, A-P 1, A-P 2$ and $A-P 3$ are not determined by the same cistron then the separate cistrons that do determine them are probably contiguous.

The modified pathogenicity phenotype of culture No. 1 on Akmolinsk (P1) can be accounted for by postulating that the parental alleles $A-P 1$ and $a-P 1$ differ by two or more base changes. Given this, recombination within these genes could produce a form of the gene unlike either of the parental alleles. Similarly the modified reaction type of cultures Nos. 2-10 on Leona (P3) suggests that the parental alleles $A-P 3$ and $a-P 3$ differ also by two or more base changes.

It has commonly been assumed that pathogenicity genes in flax rust that normally segregate as a unit are separate, closely-linked genes, although Flor (1959b) and Shepherd (1963) have both raised the possibility that they may be allelic genes. However, the allelic gene hypothesis has not been favoured because one strand can carry the genetic information necessary for the expression of all the avirulence specificities within a group. Thus, for example, if the $A-P, A-P 1, A-P 2$ and $A-P 3$ specificities are determined by a single cistron, then the product of this cistron must exist in a form that is capable of expressing (or determining) all four specificities simultaneously, 
as well as in other forms that result in no specificity, or one, two or three specificities in various combinations. In the absence of any direct evidence for such a gene product with so many apparently independent functions, the simplest assumption has been that the $A-P, A-P 1, A-P 2$ and $A-P 3$ specificities are determined by separate but closely-linked cistrons.

The frequency of variant cultures obtained in the present study does not help to decide between the alternative models. In the dikaryon-screening experiment, three variant cultures were recovered among 3160 test-cross progeny. Assuming these cultures are all recombinants, the estimated recombination frequency within the region of the $A-P, A-P 1, A-P 2$ and $A-P 3$ genes is $0 \cdot 19$ per cent given that the screening procedure is expected to detect only half of all the recombinants that arise. This value, with 95 per cent confidence limits of 0.04 per cent and 0.56 per cent, is sufficiently small to be compatible with either allelism or close-linkage of the $A-P, A-P 1$, $A-P 2$ and $A-P 3$ genes.

If the $A-P, A-P 1, A-P 2$ and $A-P 3$ specificities are determined by separate, closely-linked genes, as is commonly assumed, it should be possible to find mutant cultures that have lost just one of the avirulence specificities on a particular strand, as pointed out by Shepherd (1963). However, as described below, no such culture has been detected either in the present study or in earlier studies.

If the ten variant cultures listed in table 4 have a mutational origin, then cultures Nos. 1 and 4 would require a mutation in the $a-P A-P 1 A-P 2 A-P 3$ strand of $\mathrm{CH}_{5}$ which simultaneously modified both $A-P 1$ and $A-P 3$; the other eight cultures would require either a mutation in the $a-P A-P 1 A-P 2$ $A-P 3$ strand which resulted in the loss of all three avirulence specificities, or a mutation in the $A-P a-P 1 \quad a-P 2$ a-P3 strand which resulted in the loss of both the avirulence $A-P$ specificity as well as the semi-virulence $a-P 3$ specificity. In a screening test for somatic variants in strains $\mathrm{CH}_{5}, \mathrm{CH}_{4}$ and $\mathrm{CH}_{6}$ (described above) the only variants recovered were those which had "lost" all the avirulence specificities present in one or other of the parental strands. Similarly, the somatic variants recovered by Flor (1958) after screening X-ray treated uredospores of a race $22 \times$ race 1 hybrid on Koto $(P)$ and Leona (P3) had "lost" all the avirulence specificities in the one strand. However, as Flor suggested, the joint loss of specificities in his study could have been due to X-ray induced deletion of a segment of the pathogen chromosome. Although the somatic variants recovered in both studies could be due to somatic recombination rather than mutation, these screening tests were capable, nevertheless, of detecting mutants with only a single change in specificity, and none were found. Thus these studies do not provide supporting evidence for the assumption that the $A-P, A-P 1, A-P 2$ and $A-P 3$ specificities are determined by separate cistrons.

Finally, the results of the present study can be used to indicate the likely order of the four " $A-P$ " genes, provided that two assumptions are made. First, it is assumed that the four markers are point markers, at least in the general sense, i.e., if a marker is specified by base changes at two or more sites then a base difference specifying another marker does not fall between these sites. Second, it is assumed that the non-parental genotypes possessed by the variant cultures are each derived from meiotic recombination and not mutation. Given these assumptions, the gene order $A-P A-P 2 A-P 1 A-P 3$ permits each of the three kinds of presumptive recombinant strands 
recovered in the screening tests to be obtained by a single recombinational event, involving (i) conversion of a single marker, or coconversion of two or more contiguous markers, without reciprocal exchange (crossing-over) between parental strands, or (ii) reciprocal exchange between markers without conversion, or (iii) conversion, or coconversion of contiguous markers, with an associated reciprocal exchange.

The recombinational events from which each of the presumptive recombinant strands could have arisen, assuming the order to be $A-P A-P 2$ $A-P 1 A-P 3$, are outlined in table 5. As shown in this table the phenotypes of

\section{TABLE 5}

Observed pathogenicity phenotypes of presumptive recombinant cultures from rust strain $\mathrm{CH}_{5}$ and those expected following single recombinational events assuming $\mathrm{CH}_{5}$ has genotype A-P a-P2 a-P1 a-P3/a-P A-P2 A-P1 A-P3

Observed phenotypes of presumptive recombinants

$\begin{array}{ll}\text { Culture } & \text { Pathogenicity on host gene } \\ \text { No } & P\end{array}$

No.

1

4

$2,3,5-10$ $+\quad--+1-$ $+\quad+-+$

\section{Expected recombinant phenotypes}

\begin{tabular}{|c|c|c|c|c|}
\hline \multirow[b]{2}{*}{ Type* } & \multicolumn{4}{|c|}{ Pathogenicity on host gene } \\
\hline & $P$ & $P 1$ & $P 2$ & P3 \\
\hline A & + & -- & -- & $+1-\dagger$ \\
\hline B & + & + & -- & $+1-$ \\
\hline $\mathrm{C}$ & + & + & + & $+1-$ \\
\hline
\end{tabular}

* Phenotype A corresponds to a recombinant strand a-P A-P2 A-P1 a-P3, which could be obtained from a single recombinational event in $\mathrm{CH}_{5}$ by: (i) crossover between $A-P 1$ and $A-P 3$ or (ii) conversion of $A-P 3$ to $a-P 3$ or, (iii) conversion of $A-P \alpha-P 2 a-P 1$ to $a-P A-P 2 A-P 1$. Phenotype $\mathrm{B}$ corresponds to a recombinant strand $a-P A-P 2$ a-P1 $a-P 3$, which could be obtained from a single recombinational event in strain $\mathrm{CH}_{5}$ by: (i) crossover between $A-P 2$ and $A-P 1$ or, (ii) conversion of $A-P$ a-P2 to $a-P A-P 2$ or, (iii) conversion of $A-P 1 A-P 3$ to $a-P 1$ $a-P 3$ or, (iv) conversion of $A-P 1$ to $a-P 1$ with associated crossover between the $A-P 1 / a-P 1$ and $A-P 3 / a-P 3$ markers or, (v) conversion of $a-P 2$ to $A-P 2$ with associated crossover between the $A-P / a-P$ and $A-P 2 / a-P 2$ markers. Phenotype C corresponds to a recombinant strand $a-P$ $a-P 2 a-P 1 a-P 3$, which could be obtained from a single recombinational event in strain $\mathrm{CH}_{5}$ by: (i) crossover between $a-P$ and $A-P 2$ or, (ii) conversion of $A-P$ to $a-P$ or, (iii) conversion of $A-P 2 A-P 1 \quad A-P 3$ to $a-P 2$ a-P1 $a-P 3$ or, (iv) conversion of $A-P 2$ to $a-P 2$ with associated crossover between the $A-P 2 / a-P 2$ and $A-P 1 / a-P 1$ markers or (v) conversion of $A-P 2 A-P 1$ to $a-P 2$ a-P1 with associated crossover between the $A-P 1 / a-P 1$ and $A-P 3 / a-P 3$ markers.

$\dagger$ Based on the observation that $\mathrm{CH}_{5} \times \mathrm{I}$ progeny with the $a-P 3$ allele from parent strain $\mathrm{C}$ produced $+/-$ pathogenicity on Leona instead of the $+/-(-)$ level of growth typical of strain C (Lawrence et al., 1981).

the presumptive recombinant cultures obtained agree with those predicted from the above gene order except for the modified reactions on Akmolinsk $(P 1)$ and Leona $(P 3)$ already described. With other marker orders it is not possible to obtain all three kinds of presumptive recombinant strand by single recombinational events. However, these other orders cannot be excluded since it is known that simultaneous recombinational events within a gene, or within neighbouring cistrons, can occur as apparently separate but correlated events (for example, see Paszewski et al. 1971). If one or more of the kinds of presumptive recombinant strand obtained in the present study are derived from multiple recombinational events, then the proposed gene order may be incorrect.

In summary, the observation that a single strand can express multiple specificities in various combinations could be interpreted to mean that the 
specificities are functionally independent of each other and therefore are likely to be determined by separate cistrons. However, if this is the case, it should be possible to recover mutants that have lost only one of the avirulence specificities on a particular strand, and no such variants have been detected, either in the present study or in two somatic variation studies (Flor, 1958; Lawrence, 1977). The recombinational studies establish that the markers are sufficiently close for a common cistron to be considered a possibility, and suggest that if the markers do not belong to the same cistron then the separate cistrons that do determine them are contiguous. Clearly, conclusive evidence as to the fine-structure of these genes has not yet been obtained. The evidence presently available does suggest, however, that the common assumption that $A-P, A-P 1, A-P 2$ and $A-P 3$ are determined by separate cistrons should not be accepted without question.

Person (1959) noted that only four of the eight possible combinations of pathogenicity on Akmolinsk (P1), Abyssinian (P2) and Leona (P3) had been reported and these could be arranged to show a progressive step-wise increase in virulence range; i.e., from avirulence on all three varieties, to virulence on Akmolinsk alone, to virulence on both Akmolinsk and Abyssinian through to virulence on all three varieties. Person suggested that this pattern may be due to Akmolinsk, Abyssinian and Leona having genes $A$, $A B$ and $A B C$ (that is, $P 1, P 1 P 2$ and $P 1 P 2 P 3$ ), respectively. On this model rust stocks that attack Leona must also attack Abyssinian and Akmolinsk, and so on. However, cultures Nos. 1 and 4 (table 4) both have reaction patterns that differ from the four reported above and are therefore inconsistent with Person's hypothesis. It may be simply due to chance sampling that only the four reaction patterns described above have been found in rust strains collected from the wild.

\section{(ii) Origin of variant pustules resembling strain $I$}

The three distinctive features of the pustules listed under $4 \mathrm{~b}$ in table 1 are that they are apparently derived from strain I alone, that it is likely that they are each derived from a separate event and that they were recovered in unequal numbers on the different tester-host stocks. As noted earlier, this third feature suggests that these pustules did not arise from virulent spores present in the original inoculum since the tester-host stocks are apparently equally efficient at screening out rare virulent spores present in an inoculum of avirulent spores. Therefore these pustules could be derived from rare cells possessing two strain I nuclei that arose during the small amount of growth avirulent $\mathrm{CH}_{5} \times \mathrm{I}$ test-cross aeciospores can undergo on the testerhost stocks before the onset of the hypersensitive reaction.

Two possible mechanisms could produce occasional hyphal cells possessing two strain I nuclei instead of the usual combination of one $\mathrm{CH}_{5}$ and one I nucleus. First, in the process of aeciospore formation occasional tri-nucleate spores may have been produced with one $\mathrm{CH}_{5}$ nucleus and two I nuclei. Such spores on germination could produce cells possessing only the two strain I nuclei. Alternatively, hyphae from different avirulent $\mathrm{CH}_{5} \times \mathrm{I}$ aeciospores may have fused occasionally and exchanged nuclei during the short period of growth prior to the hypersensitive reaction in the tester hosts. In this way a cell possessing two strain I nuclei of different mating type could have been produced, since each inoculum consisted of $\mathrm{CH}_{5} \times \mathrm{I}$ aeciospores 
derived from many separate crosses. Flor (1964) has shown that two different strains of rust growing together on a susceptible host can exchange nuclei in this way. Both of these mechanisms will result in rare virulent pustules, each derived from a separate event, and with a genotype equivalent to strain I selfed. On this basis the pustules are expected to show different pathogenicity patterns on some of the differential varieties due to segregation, and to be all avirulent on Bolley Golden selection, and the type $4 \mathrm{~b}$ pustules showed such behaviour.

The problem remains of accounting for the markedly different frequency of occurrence of pustules of this type on the three tester-host stocks. One possibility is that the amount of growth made by avirulent spores before the hypersensitive reaction is established may not be the same on the different tester stocks. If this is so, then rare virulent cells might be expected to arise most frequently on the tester host on which the growth was most extensive, because more extensive growth should increase the opportunities for nuclear reassortment or exchange to occur. A second possibility is that the rare strain I type cells arise with the same frequency on the different tester hosts, but that not all of them subsequently succeed in making sufficient growth to form a pustule, and that the proportion that do succeed varies from host to host. The failure of a newly formed virulent cell to produce a pustule could be due to the hypersensitive reaction associated with the avirulent hyphae from which it arose. If the severity of the hypersensitive reaction differs from host to host then the probability of a virulent cell achieving growth may not be the same on all host varieties.

Acknowledgements. - We express our appreciation to the Australian Research Grants Committee for financial support. G.J.L. was supported by a Commonwealth Postgraduate Research Award. We thank Dr Jean Mayo for useful discussions.

\section{REFERENCES}

DINOOR, A., KHAIR, J., AND FLeISCHMANN, G. 1968. Pathogenic variability and the unit representing a single fertilization in Puccinia coronata var, avenae. Canad. J, Bot, , 46, $501-508$.

FLOR, H. H. 1955. Host-parasite interaction in flax rust-its genetics and other implications. Phytopath., 45, 680-685.

FLOR, H. H. 1956. The complementary genic systems in flax and flax rust. Adv. Genet., 8, 29-54.

FLOR, H. H. 1958. Mutation to wider virulence in Melampsora lini. Phytopath., 48, 297-301.

FLOR, H. H. 1959a. Differential host range of the monokaryon and dikaryons of a euautoecious rust. Phytopath., 49, 794-795.

FLOR, H. H. 1959b. Genetic controls of host-parasite interactions in rust diseases. In Plant Pathology-Problems and Progress 1908-1958, ed. C. S. Holton, pp. 137-144. University of Wisconsin Press.

FLOR, H. H. 1964. Genetics of somatic variation for pathogenicity in Melampsora lini. Phytopath., 54, 823-826.

FLOR, H. H. 1965a. Inheritance of smooth-spore-wall and pathogenicity in Melampsora lini. Phytopath., 55, 724-727.

FLOR, H. H. $1965 b$. Tests for allelism of rust-resistance genes in flax. Crop Sci., 5, 415-418.

LAWRENCE, G. J. 1977. Genetics of pathogenicity in flax rust. Ph.D. thesis, University of Adelaide.

LAWRENCE, G. J. 1980. Multiple mating-type specificities in the fiax rust Melampsora lini. Science, 209, $501-503$.

LAWRENCE, G. J., MAYO, G. M. E., AND SHEPHERD, K. W. 1981. Interactions between genes controlling pathogenicity in the flax rust fungus. Phytopath, 71,12-19. 
MAYO, G. M. E., AND SHEPHERD, K. W. 1980. Studies of genes controlling specific hostparasite interactions in flax and its rust. 1. Fine structure analysis of the $M$ group in the host. Heredity, 44, 211-227.

PASZEWSKI, A., PRAZMO, W., AND JASZCZUK, E. 1971. Multiple recombinational events within the $84 w$ locus of Ascobolus immersus. Genet. Res., Camb., 18, 199-214.

PERSON, C. 1959. Gene-for-gene relationships in host-parasite systems. Can. J. Bot., 37, 1101-1130.

SHEPHERD, K. W. 1963. Genetics of host-pathogen interactions. Ph.D. thesis, University of Adelaide.

SHEPHERD, K. W., AND MAYO, G. M. E. 1972. Genes conferring specific plant disease resistance. Science, 175, 375-380.

STATLER, G. D., AND GOLD, R. E. 1980. Comparative virulence of basidiospore and urediospore cultures of three pathogenic races of Melampsora lini. Phytopath., 70, 555557. 